\title{
Chronic Inflammatory Demyelinating Polyneuropathy Due to the Administration of Pegylated Interferon $\alpha-2 b$ : A Neuropathology Case Report
}

\author{
Kensuke Shiga ${ }^{1}$, Eijiroh Tanaka ${ }^{1}$, Reina Isayama ${ }^{1}$, Toshiki Mizuno ${ }^{1}$, \\ Kyoko Itoh $^{2}$ and Masanori Nakagawa ${ }^{1}$
}

\begin{abstract}
We report a 35-year-old man who developed weakness in his extremities five months after pegylated interferon $\alpha$ (IFN $\alpha)$-2b was administered. The serum tumor necrosis factor- $\alpha$ (TNF $\alpha)$ was elevated and nerve conduction studies revealed demyelination both in the distal and intermediate segments. The sural nerve pathology showed mild demyelinating process. The cessation of IFN $\alpha$ and administration of intravenous immunoglobulin improved both his clinical symptoms and the temporal dispersion in motor nerve conduction study. IFN $\alpha$-induced CIDP is presumably a transient immunological condition that requires immunomodulatory therapy. The elevated serum TNF $\alpha$ may implicate the degree of downstream autoimmunitiy induced by IFN $\alpha$.
\end{abstract}

Key words: chronic inflammatory demyelinating polyneuropathy, interferon $\alpha-2 b$, adverse effect, immunoglobulin

(Intern Med 51: 217-221, 2012)

(DOI: 10.2169/internalmedicine.51.6320)

\section{Introduction}

Interferons comprise an evolutionarily conserved family of secreted proteins that participate as extracellular messengers in a variety of responses, including antiviral, antiproliferative, and immunomodulatory properties that maintain host defense systems and homeostasis (1). The administration of interferon alpha (IFN $\alpha$ ) is commonly used as the first-line therapy for patients with chronic viral hepatitis because of its antiviral effects (2). On the other hand, autoimmunity is a well-recognized complication of IFN $\alpha$ therapy, with reported frequencies ranging between $4.3 \%$ and $18.5 \%$ in large observational studies (3-5). Neuropathies due to administration of IFN $\alpha$ are a rare complication $(3,4)$; however, eight distinct case reports have shown that chronic inflammatory demyelinating polyneuropathy (CIDP) can result from administration of IFN $\alpha$, presumably due to perturbations in the host immune system (6-13). We here report a biopsy-proven case with IFN $\alpha$-induced CIDP in which the serum value of tumor necrosis factor- $\alpha(\mathrm{TNF} \alpha)$ was elevated. The correlation between the anatomical distribution of demyelination and the serum value of TNF $\alpha$ was discussed.

\section{Case Report}

A 35-year-old man was admitted to our facility because of progressive weakness and numbness in his extremities five months after the antiviral therapy was initiated to treat chronic type $\mathrm{C}$ hepatitis using pegIFN $\alpha-2 b$ and ribavirin. At first, he felt subtle numbness in his toes and fingers. Two months later, the tingling sensations gradually spread to his soles and palms, and he began to have difficulty climbing upstairs. In the following month, he became unable to screw caps of bottles or button up his shirts. Five months after the beginning of his neurological symptoms he was referred to our department, at which point the referred neurologist discontinued pegIFN $\alpha-2 b$ and ribavirin due to the possible ad-

${ }^{1}$ Department of Neurology, Kyoto Prefectural University of Medicine, Japan and ${ }^{2}$ Department of Molecular Pathology, Kyoto Prefectural University of Medicine, Japan

Received for publication August 3, 2011; Accepted for publication October 7, 2011

Correspondence to Dr. Kensuke Shiga, kenshiga@koto.kpu-m.ac.jp 
Table 1. Summary of Nerve Conduction Studies on Admission

\begin{tabular}{|c|c|c|c|c|}
\hline & left median & left ulnar & left tibial & \\
\hline$\overline{\mathrm{DL}}(\mathrm{ms})$ & $5.2 *(<4.2)$ & $4.4^{*}(<3.4)$ & $4.8(<6.0)$ & \\
\hline CMAP (mV) & $4.4(>3.5)$ & $4.8(>2.7)$ & $5.7(>2.9)$ & \\
\hline $\operatorname{MCV}(\mathrm{m} / \mathrm{s})$ & $24.7 *(>48)$ & $33.6^{*}(>49)$ & $43.6(>41)$ & \\
\hline min F-latency (ms) & $54.8 *(<31)$ & $49.2 *(<32)$ & $70.8 *(<58)$ & \\
\hline F occurrence $(\%)$ & 56.0 & 50.0 & $43.6^{*}(>93)$ & \\
\hline & left median & left ulnar & left sural & right sural \\
\hline SNAP $(\mu \mathrm{V})$ & n.e.* $(>19)$ & $1.8^{*}(>19)$ & $10.9 \quad(>8.3)$ & $15.3 \quad(>8.3)$ \\
\hline $\mathrm{SCV}(\mathrm{m} / \mathrm{s})$ & $(<47)$ & $19.2^{*}(<44)$ & $53.1 \quad(<48)$ & $55.1 \quad(<48)$ \\
\hline
\end{tabular}

DL: distal latency, CMAP: compound muscle action potential, MCV: motor conduction velocity, SNAP: sensory nerve action potential, SCV: sensory conduction velocity. MCVs were calculated between wrist and elbow in the median and ulnar nerves and between ankle and popliteal fossa in the tibial nerve. SCVs were measured between index finger and wrist in the median nerve and between ring finger and wrist in the ulnar nerve. The sural SCV was calculated between posterior calf and the lateral foot. Asterisks indicate abnormal values. n.e.: not elicited. The values in parentheses indicate normal value in each parameter in our facility.

verse side effects. However, the cessation of pegIFN $\alpha-2 b$ did not alleviate the weakness or the numbness in his extremities, and he was admitted to our facility. On neurological examination, the patient was alert and cranial nerves were intact. His muscle bulk was preserved and no fasciculation was observed. His Medical Research Council (MRC) scores were as follows: deltoid, 5; biceps brachii, 4; triceps brachii, 5; wrist extensors and flexors, 4; iliopsoas, 5; quadriceps femoris, 5; tibialis anterior, 3; extensor hallucis longus, 3. The patient complained of paresthesia in a stockingand-glove distribution. Pinprick and vibration sensation were decreased in his toes and fingers. The deep tendon reflexes were diminished. Bilateral plantar reflexes were flexor. Neither orthostatic hypotension nor dysuria was evident.

The urine analysis, the complete blood count and the biochemical analysis were normal. Serum M-protein was negative by means of immunoelectrophoresis. Also, serum antibodies against glycolipids, including GM1, GM2, GM3, GA1, GD1b, GD3, GT1b, GQ1b, and galactocerebroside-C were negative. The antibody against $\alpha-2 b$ was negative. It is noteworthy serum tumor necrosis factor- $\alpha$ was elevated at $9.2 \mathrm{pg} / \mathrm{mL}$ (normal value; $0.6-2.8 \mathrm{pg} / \mathrm{mL}$ ). The protein level in the cerebrospinal fluid (CSF) was slightly elevated at 50 $\mathrm{mg} / \mathrm{dL}$, while the number of CSF cells was $3 / \mathrm{mm}^{3}$. The results of the nerve conduction studies are shown in Table 1. In the motor nerve conduction studies, the distal motor latencies were prolonged; $5.2 \mathrm{~ms}$ and $4.4 \mathrm{~ms}$ in the left median and left ulnar nerves, respectively. The motor nerve conduction velocities were markedly reduced; $24.7 \mathrm{~m} / \mathrm{s}$ and $33.6 \mathrm{~m} / \mathrm{s}$, in the left median and in the left ulnar nerve, respectively. One notable finding was a temporal dispersion of the compound muscle action potentials (CMAPs) of the left median nerve at the elbow, while the distal CMAP was relatively unaffected following stimulation at the wrist (Fig. 1A). A similar dispersion pattern was also observed in the right median and the left tibial nerves (data not shown). The sensory nerve action potentials (SNAPs) were not elicited or were only barely elicited in the left median and left ulnar nerves, respectively, whereas the SNAP in the right sural nerve and that in the right counterpart was $15.3 \mu \mathrm{V}$ and $10.9 \mu \mathrm{V}$, respectively. Over the following three weeks, the SNAP in the right sural nerve was reduced from 15.3 $\mu \mathrm{V}$ to $3.6 \mu \mathrm{V}$. A nerve biopsy was then performed on the right sural nerve and the teased fiber analysis revealed mild demyelinating pathology, including myelin wrinkling, segmental demyelination and remyelination (Fig. 2A). On toluidine blue-stained, semi-thin sections, the number of large myelinated fibers was slightly decreased and variably thinned myelin sheaths were scattered (Fig. 2B, thin arrows). In contrast, both the axons and unmyelinated nerve fibers were relatively preserved. Invasion of macrophages into the endoneurium was also demonstrated (Fig. 2B, thick arrow). A myelinated fiber (Fig. 2C, arrow) showed electron-dense materials which looked like lysosomes (Fig. 2C, small arrowhead, inset) situated in the Schwann cytoplasm at the node of Ranvier. Another myelinated fiber showed lamellated body in the Schwann cytoplasm (Fig. 2C, large arrowhead). Axons were well preserved in myelinated fibers (Fig. 2C, asterisks). The results of the nerve conduction and the pathological evidence of mild demyelination led us to diagnose him as having CIDP.

To determine any possible improvement after the cessation of pegIFN $\alpha-2 b$, the patient was observed for three weeks without any additional treatment; however, the weakness in the patient's legs further deteriorated. High-dose intravenous immunoglobulin (IVIG; $0.4 \mathrm{mg} / \mathrm{kg} /$ day for five consecutive days) was then administered. Within a week after the administration of IVIG, both the averaged hand grip and the neuropathy disability scores (NDS) (14) recovered to a remarkable extent; however, weakness in the extremities started to deteriorate in three weeks. Two additional courses of IVIG were administered during the following two months; the patient's neurological status showed marked improvement within a week following each administration (Fig. 3). The fourth administration of IVIG was canceled due to a moderate elevation in the levels of liver enzymes, probably an adverse effect of immunoglobulin administration. Fortunately, both the hand grip and NDS showed a 
A

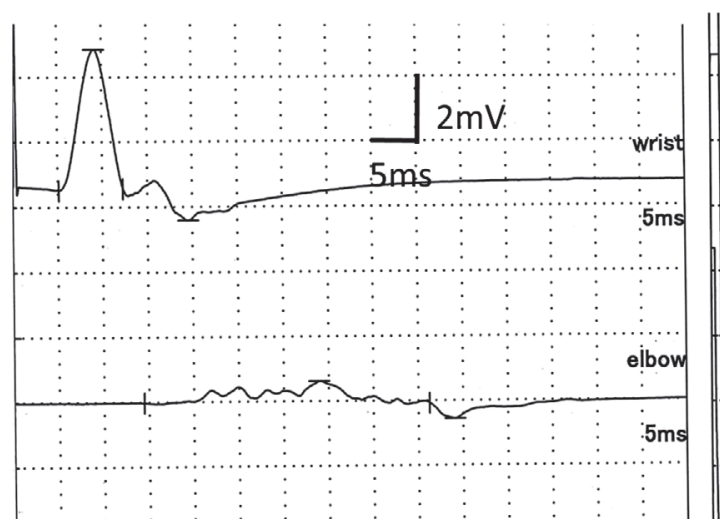

B

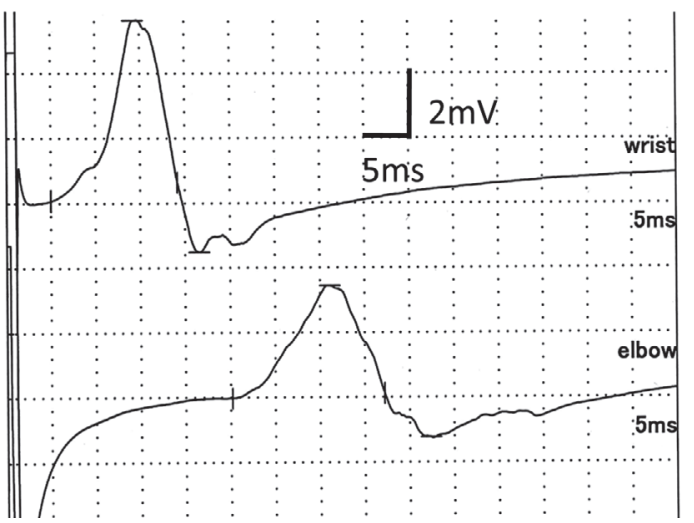

Figure 1. A. Results of the motor conduction study performed on the left median nerve upon admission (five months after onset). The compound muscle action potentials (CMAP) elicited at the elbow shows a marked temporal dispersion, suggesting uneven demyelination between the wrist and elbow. The dispersion pattern and duration of the CMAP was almost the same at the axilla. B. Results of the motor nerve conduction study performed on the left median nerve after three courses of high-dose IVIG therapy (eight months after onset). The CMAP elicited at the elbow shows marked improvement in terms of the dispersion pattern, although the latency period is prolonged compared to Fig. $1 \mathrm{~A}$, which is suggestive of a remyelination process.
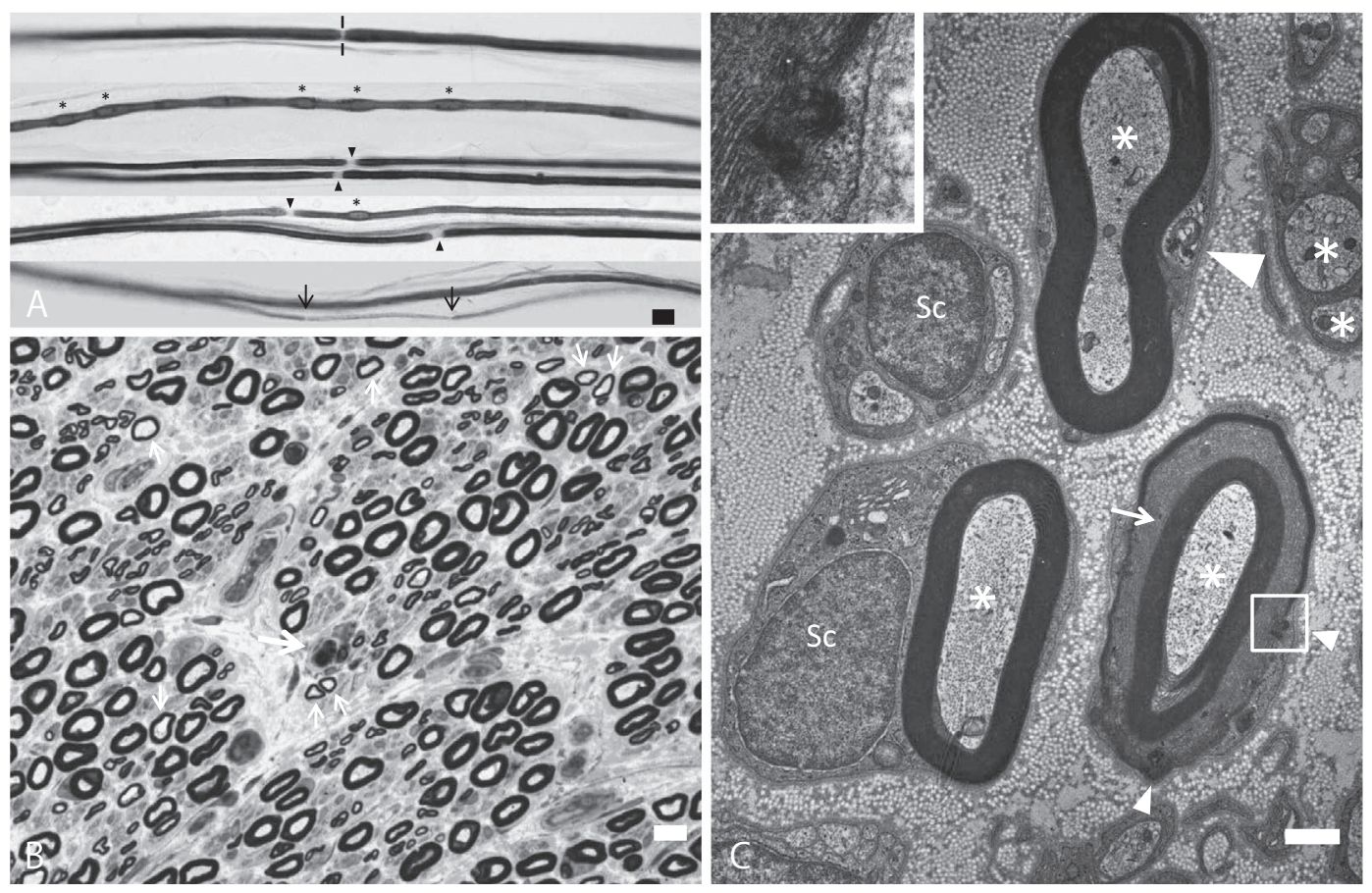

Figure 2. A. Teased fiber specimen. The pair of short longitudinal lines (top) indicates the normal hiatus of Ranvier node. The asterisks indicate wrinkling of myelin. The small arrowheads indicate segmental demyelinations or the widening of Ranvier node. The arrows on the nerve (bottom) indicate the shortening of the intermodal segment of the remyelinated nerve. Bar $=10 \mu \mathrm{m}$. B. Sections of the sural nerve showing a large number of variably thinned myelin sheaths (thin arrows) and macrophages invading into the endoneurium (thick arrow). Toluidine blue-stained semi-thin section. Bar=10 $\mu \mathrm{m}$. C. At the Schmidt-Lanterman incisure (arrow), irregularly shaped electron-dense materials (small arrowhead) were found in the cytoplasm of the Schwann cells (Sc). Other Sc show electron-dense granules and myelin-like materials in the cytoplasm (large arrowhead). The axons were well preserved in both the myelinated and unmyelinated fibers (*). Bar $=2 \mu \mathrm{m}$. 


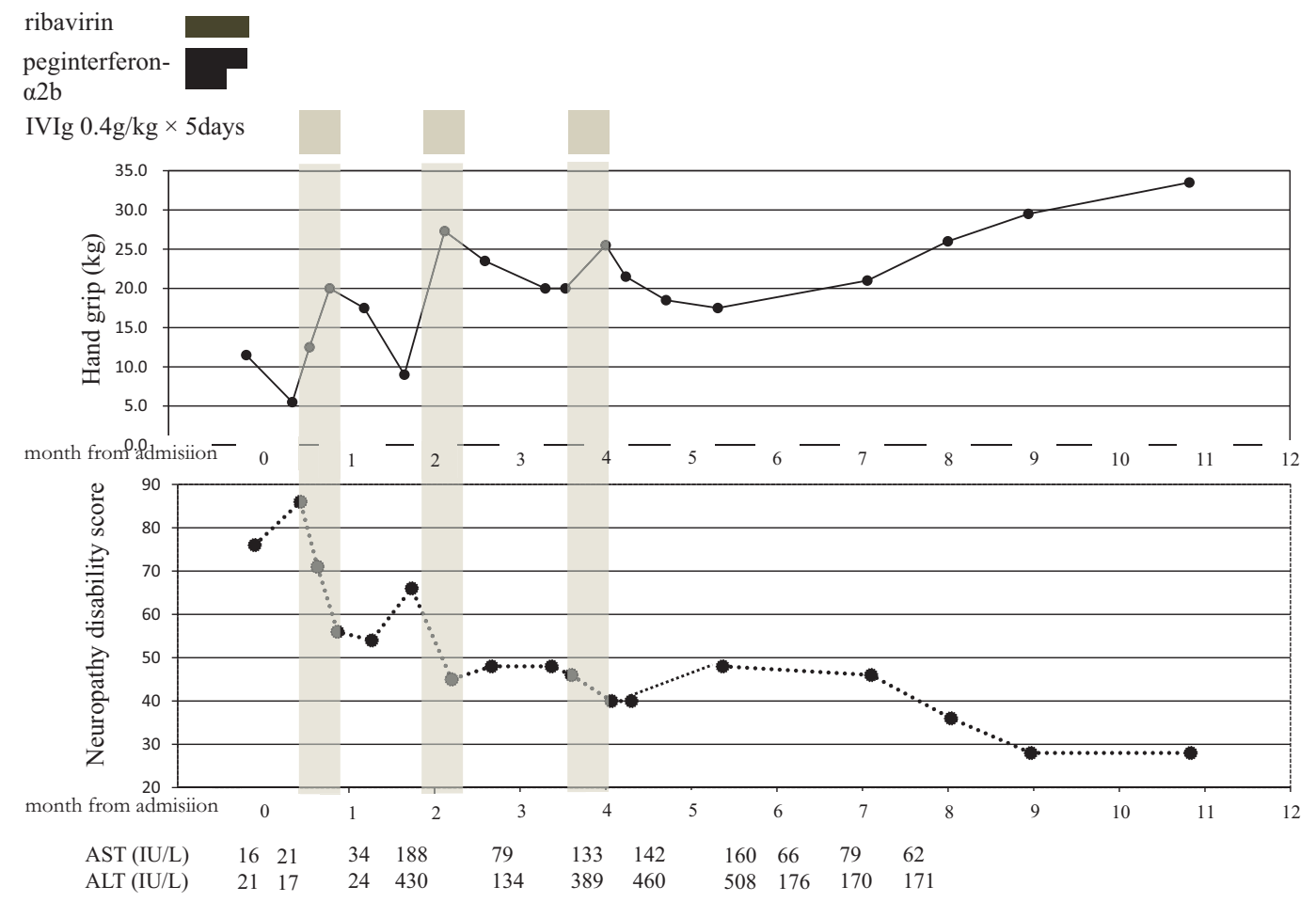

Figure 3. The clinical course of the patient based on two neurological indices that were measured over the course of one year; average hand grip and neuropathy disability scores (NDS). Each course of high-dose intravenous immunoglobulin therapy (IVIG) improved both the average hand grip and NDS. After the third course of IVIG, the indices gradually improved over the ensuing months. The patient's neurological status has been stable for three years (data not shown).

gradual improvement without further administration of IVIG (Fig. 3). Follow-up NCS revealed improved synchronization of the CMAP at the elbow (Fig. 1B), indicating a remyelination process at this segment. The patient did not require further maintenance therapy, such as oral prednisone or immunosuppressants, and he has been free of neurological symptoms for three years.

\section{Discussion}

We reported the clinical characteristics of a patient with CIDP whose symptoms started one month after the administration of pegIFN $\alpha-2 b$ and gradually worsened over the subsequent five months. The patient's neurological status did not improve after the discontinuation of pegIFN $\alpha-2 b$, whereas the subsequent administration of IVIG resulted in rapid improvement of the patient's motor weaknesses. Two additional courses of IVIG were required during the following months; however, no maintenance therapy was needed for three years.

The results of nerve conduction studies indicated that the demyelinating process involved both the distal and the intermediate segments of the nerve trunks for the following reasons. First, the sensory nerve conduction study revealed decreased SNAPs in the median and the ulnar nerves and a relatively unaffected SNAP in the sural nerves: this pattern, called an abnormal median and normal sural sensory response (AMNSSR) pattern, indicates demyelination in the distal terminals of sensory nerves $(15,16)$. Secondly, the motor nerve conduction study showed temporal dispersion within the intermediate segments of the median (Fig. 1A) and tibial nerves, indicating that the demyelination process also involved the intermediate nerve segments. In other words, the demyelination occurred in both the distal and intermediate segments; thus, the condition was classified as the diffuse type, according to the regional classification of CIDP (15).

Kuwabara et al reported that the serum value of tumor necrosis factor (TNF)- $\alpha$ is elevated only in patients with the diffuse type, among the three regional variatns of CIDP (15). In the patient described here, the serum TNF- $\alpha$ value was three fold the upper limit of the normal value. TNF- $\alpha$, a proinflammatory cytokine, is secreted from T-cells and macrophages and exerts toxic effects on peripheral myelin and endothelial cells (17). The sural nerve pathology of this patient indicated extravasation of macrophages into the endoneurium (Fig. 2B), which is possible evidence of breakdown of the BNB and activation of macrophages within the intermediate nerve segment. It can therefore be speculated that exogenous IFN $\alpha$ might have induced the release of adhesion molecules or cytokines, such as TNF- $\alpha$, leading to the possible breakdown of the $\mathrm{BNB}$ and migration of macrophages into the endoneurium.

In studies on IFN $\alpha$-related complications, therapy-related neuropathies were reported in only 3 of 11,241 patients with hepatitis C in one study (3) and in 0 of 987 patients in an- 
other study (4); thus, therapy-related neuropathies are considered a rare complication of IFN $\alpha$ treatment. Nevertheless, seven distinct cases of CIDP have been reported after the administration of a variety of IFN $\alpha$ species, including IFN $\alpha$ (6-9), pegIFN $\alpha-2 \mathrm{a}(10)$, and pegIFN $\alpha-2 b(11,12)$. The interval between the administration of IFN $\alpha$ and the onset of CIDP ranged from 3 weeks to 11 months. Among these cases, three cases were observed following the absence of therapeutic intervention for up to 6 weeks $(6,7,11)$; however, the neurologic symptoms deteriorated in all of these cases, similar to the present patient. It seems that once a causative immunologic trigger is initiated, the pathologic process leading to demyelination is not interrupted even if IFN $\alpha$ is discontinued. Autoimmunity, once induced by IFN $\alpha$, may trigger multiple downstream mechanisms, including the increased expression of MHC class I antigens and stimulation of the transcription of cytokines (including TNF- $\alpha$ ), leading to the activation of lymphocytes, macrophages, and natural killer cells (2). Among the six reported cases of IFN- $\alpha$-induced CIDP, three cases were classified as the diffuse type and three cases the intermediate type, suggesting the breakdown of BNB within the intermediate segments in IFN- $\alpha$-induced CIDP and possible involvement of TNF- $\alpha$ in the pathogenesis of demyelination.

On the other hand, however, a favorable outcome was reported in all of the reported cases of IFN $\alpha$-induced CIDP when the patients were treated with any of the standard therapies for CIDP, including oral steroids $(6,8)$, plasma exchange $(7,11)$, or IVIG $(8-10,12)$. In the present case, three courses of IVIG were administered, but no maintenance immunomodulatory therapy was needed. In the reported cases of IFN $\alpha$-induced CIDP, a few of relapses occurred; however, no single case required the administration of prednisone or immunosuppressants. These features imply that IFN $\alpha$-induced CIDP is a transient condition that requires a certain period of immunomodulatory therapy, but does not require the administration of a long-term maintenance therapy.

\section{Conclusion}

Here, the clinical characteristics of a patient with CIDP due to administration of pegIFN $\alpha-2 b$ are reported. IFN $\alpha-$ induced CIDP is, in general, a benign and transient condition; however, immunomodulatory therapy is required to reverse the demyelinating process once it develops. The measurement of the serum TNF $\alpha$ may be useful to assess the degree of autoimmunity in IFN $\alpha$-induced CIDP.

The authors state that they have no Conflict of Interest (COI).

\section{Acknowledgement}

The authors would like to thank Prof. Kusunoki (Department of Neurology, Kinki University, Osaka, Japan) for measuring the serum values of the glycolipid antibodies.

\section{References}

1. Dusheiko GM. Interferon $\alpha$ : Biology, Pharmacology and Therapy for Chronic Viral Hepatitis. In: The Cytokine Handbook. Thompson AW, Lotze MT, Eds. Academic Press, Amsterdam, 2003: 1233-1254.

2. Stübgen JP. Interferon alpha and neuromuscular disorders. J Neuroimmunol 207: 3-17, 2009.

3. Fattovich G, Giustina G, Favarato S, Ruol A. A survey of adverse events in 11,241 patients with chronic viral hepatitis treated with alpha interferon. J Hepatol 24: 24-38, 1996.

4. Okanoue T, Sakamaoto S, Itoh Y, et al. Side effects of high dose interferon therapy for chronic hepatitis C. J Hepatol 25: 283-291, 1996.

5. Rönnbolm LE, Alm GV, Oberg KE. Autoimmunity after alphainterferon therapy for malignant carcinoid tumors. Ann Intern Med 115: 178-183, 1991.

6. Marzo ME, Tintoré M, Fabregues O, Montalbán X, Codina A. Chronic inflammatory demyelinating polyneuropathy during treatment with interferon- $\alpha$. J Neurol Neurosurg Psychiatry 65: 604615, 1998.

7. Meriggioli M, Rowin J. Chronic inflammatory demyelinating polyneuropathy after treatment of interferon- $\alpha$. Muscle Nerve 23: 433-435, 2000.

8. Anthoney DA, Bone I, Evans TR. Inflammatory demyelinating polyneuropathy: A complication of immunotherapy in malignant melanoma. Ann Oncol 11: 1197-1200, 2000.

9. Hirotani M, Nakano H, Ura S, et al. Chronic inflammatory demyelinating polyneuropathy after treatment with interferon-alpha. Intern Med 48: 373-375, 2009.

10. Khiani V, Kelly T, Shibli A, Jensen D, Nohanty SR. Acute inflammatory demyelinating polyneuropathy associated with pegylated inferferon $\alpha 2$ a therapy for chronic hepatitis $\mathrm{C}$ virus infection. World J Gastroenterol 14: 318-321, 2008.

11. Kato-Motozaki Y, Komai K, Takahashi K, et al. Polyethylene glycol interferon $\alpha$-2b-induced immune-mediated polyradiculoneuropathy. Intern Med 48: 569-572, 2009.

12. Bassetti BR, Sturzeneker T, Ciriaco JGM, Neto LESP. Chronic inflammatory demyelinating poluneuropathy after treatment with pegylated interferon alpha $2 \mathrm{~b}$ in a patient with $\mathrm{HIV} / \mathrm{HCV}$ coinfection: case report [Article in Portugese]. Rev Soc Bras Med Trop 43: 89-91, 2010 (in Portugese).

13. Chang Ying, Han Xue-mei, Gao Yao, Liu Song-Yan. Chronic inflammatory demyelinating polyneuropathy following treatment with peglated intereferon $\alpha-2$ a for chronic hepatitis $C$ virus infection. Neurol India 59: 141-142, 2011.

14. Dyck PJ, O'Brien PC, Oviatt KF, et al. Prednisone improves chronic inflammatory demylinating polyneuropathy more than no treatment. Ann Neurol 11: 136-141, 1982.

15. Kuwabara S, Ogawara K, Misawa S, Mori M, Hattori T. Distribution patterns of demyelination correlate with clinical profiles in chronic inflammatory demyelinating polyneuropathy. J Neurol Neurosurg Psychiatry 72: 37-42, 2002.

16. Bromberg MB, Albers JW. Patterns of sensory nerve conduction abnormalities in demyelinating and axonal peripheral disorders. Muscle Nerve 16: 262-266, 2003.

17. Uncini A, Di Muzio A, Di Guglielmo G, et al. Effect of rh TNF- $\alpha$ injection into rat schiatic nerve. J Immunol 95: 88-94, 1999.

(C) 2012 The Japanese Society of Internal Medicine http://www.naika.or.jp/imindex.html 